\title{
Modelling and Simulation of a Packed Bed of Pulp Fibers Using Mixed Collocation Method
}

\author{
Ishfaq Ahmad Ganaie, ${ }^{1}$ Shelly Arora, ${ }^{2}$ and V. K. Kukreja ${ }^{1}$ \\ ${ }^{1}$ Department of Mathematics, SLIET, Longowal, Punjab 148106, India \\ ${ }^{2}$ Department of Mathematics, Punjabi University, Patiala, Punjab 147002, India
}

Correspondence should be addressed to V. K. Kukreja; vkkukreja@gmail.com

Received 30 April 2013; Revised 13 August 2013; Accepted 13 August 2013

Academic Editor: D. D. Ganji

Copyright ( 2013 Ishfaq Ahmad Ganaie et al. This is an open access article distributed under the Creative Commons Attribution License, which permits unrestricted use, distribution, and reproduction in any medium, provided the original work is properly cited.

\begin{abstract}
A convenient computational approach for solving mathematical model related to diffusion dispersion during flow through packed bed is presented. The algorithm is based on the mixed collocation method. The method is particularly useful for solving stiff system arising in chemical and process engineering. The convergence of the method is found to be of order 2 using the roots of shifted Chebyshev polynomial. Model is verified using the literature data. This method has provided a convenient check on the accuracy of the results for wide range of parameters, namely, Peclet numbers. Breakthrough curves are plotted to check the effect of Peclet number on average and exit solute concentrations.
\end{abstract}

\section{Introduction}

The removal of the soluble material, occupying the interstitial space between the particles and in the pores of the particles, from a saturated packed bed of particles is carried out by the introduction of a solvent, for example, water or weak wash liquor flowing through the bed. Solute removal is associated with diffusion like dispersion of the solvent in the direction of flow, known as longitudinal dispersion. The mechanics involved are the sum of displacement of the fluid containing solute by movement of water plug controlled by fluid mechanics, dispersion due to back mixing, diffusion due to concentration gradient, and adsorption-desorption due to relative affinity of various solutes towards the particle surface. The core problem is the prediction of the behavior of initially sharp interface between the liquids having identical dynamical and kinematical properties. This problem is of considerable practical importance, for example, in determining the efficiency of solvent utilization or filtrate recovery in washing of filter cakes. Mathematically, such chemical engineering processes can be best described by two point boundary value problems as follows:

$$
a_{1} \frac{\partial^{2} c}{\partial \xi^{2}}+a_{2} \frac{\partial c}{\partial \xi}=a_{3} \frac{\partial c}{\partial \tau}+a_{4} \frac{\partial q}{\partial \tau}, \quad(\xi, \tau) \in D
$$

where $D=\Omega \times(0, T]=(a, b) \times(0, T]$, along with the boundary conditions as follows:

$$
\begin{array}{ll}
b_{1} c+b_{2} \frac{\partial c}{\partial \xi}=k_{1} & \text { at } \xi=a, \\
b_{3} c+b_{4} \frac{\partial c}{\partial \xi}=k_{2} & \text { at } \xi=b .
\end{array}
$$

A great deal of effort has been applied to compute efficiently the solution of transient partial differential equations (1) and (2) analytically [1-7] and numerical algorithms such as Hermite radial basis function interpolation numerical scheme [8], two-stage Lie-group shooting method [9], finite difference method [10-12], spectral collocation method [13], Sinc differential quadrature method [14], orthogonal collocation method [10,15-17], fitted mesh collocation method [18], a novel numerical scheme [19], Galerkin/Petrov Galerkin method [20-22], orthogonal collocation on finite elements [23-25], factorized diagonal Padé approximation [26], spline collocation methods $[27,28]$, variational iteration method 


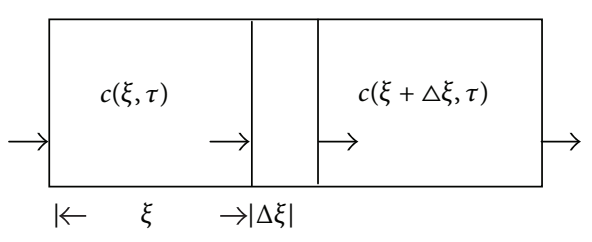

Figure 1: A simple shell balance.

(VIM) [29, 30], homotopy analysis method [31, 32], homotopy perturbation method (HPM) [33-35], and energy balance method [36].

In finite-difference method, however, the solution of the system is very unstable and requires strict selection of step size. Nevertheless, the accuracy of numerical solution is not so high. The discretization of even a few PDEs by the method of lines can lead to an extremely large system of ODEs; the numerical solution of which may have severe cost and storage implications. The Galerkin method is unsuitable for large systems because the integration process becomes very tedious. The method of OCM here is preferred over HPM and VIM, due to its simplicity and easy adaptability to computer codes. In OCM the choice of trial function is comparatively simple over other weighted residual methods. Due to its easy adaptability to computer codes, Finlayson [37] has also preferred OCM over other weighted residual methods and also over HPM and VIM.

The present paper emphasizes the use of mixed collocation method. It is a variation of the weighted residual method. It was developed to solve the models related to the transport phenomenon by Villadsen and Stewart [15]. The collocation points are the zeros of the orthogonal polynomials taken from the expansion of the trial function, enforcing the residual to vanish at the collocation points. It is a very simple, elegant, and particularly useful technique for chemical engineering problems. It gained popularity due to less computational time and easy adaptability to the computer programs. Its accuracy is comparable with the analytical one.

\section{Theoretical Formulation}

Consider a thin slice of a packed bed consisting of homogeneous symmetrical porous particles, as shown in Figure 1, through which filtrate or wash water flows. The mass balance equation for the packed bed can be described by an axial dispersion model as

$$
D_{L} \frac{\partial^{2} c}{\partial \xi^{2}}=u \frac{\partial c}{\partial \xi}+\frac{\partial c}{\partial \tau}+\frac{1-\varepsilon}{\varepsilon} \frac{\partial q}{\partial \tau}
$$

The adsorption equilibrium relationship, describing the intraparticle solute concentration as a function of the external solute concentration, is linear; that is, $q=k c$. The inlet boundary condition is assumed to be Danckwerts as

$$
u c-D_{L} \frac{\partial c}{\partial \xi}=u C_{S}, \quad \text { at } \xi=0, \forall \tau \geq 0
$$

At the outlet, no flux is assumed; that is, there is no loss of solute from the bed through the plane at which the displacing liquid is introduced as follows:

$$
\frac{\partial c}{\partial \xi}=0, \quad \text { at } \xi=L, \forall \tau \quad 0 .
$$

The initial condition is

$$
c=q=C_{i}, \quad \text { at } \tau=0, \forall \xi .
$$

The equations (3)-(6) are converted into the dimensionless form using dimensionless parameters mentioned in nomenclature. The following equations are obtained:

$$
\begin{gathered}
\frac{1}{\operatorname{Pe}} \frac{\partial^{2} C}{\partial \xi^{* 2}}=\frac{\partial C}{\partial \xi^{*}}+\frac{\varepsilon}{\varepsilon+(1-\varepsilon) k} \frac{\partial C}{\partial \tau^{*}}+\frac{1-\varepsilon}{\varepsilon+(1-\varepsilon) k} \frac{\partial Q}{\partial \tau^{*}} \\
C-\frac{1}{\operatorname{Pe}} \frac{\partial C}{\partial \xi^{*}}=0, \quad \text { at } \xi^{*}=0, \forall \tau^{*} \geq 0 ; \\
\frac{\partial C}{\partial \xi^{*}}=0, \quad \text { at } \xi^{*}=1, \forall \tau^{*} \geq 0 ; \\
C=Q=1, \quad \text { at } \tau^{*}=0, \forall \xi^{*} .
\end{gathered}
$$

The exit solute concentration $\left(C_{e}\right)$ is calculated at $\xi^{*}=1$. The average solute concentration $\left(C_{\mathrm{av}}\right)$ is calculated by the integration over the bed cross section as

$$
C_{\mathrm{av}}=\int_{0}^{1} C d \xi^{*}
$$

\section{Numerical Procedure}

Details about the collocation method are available elsewhere $[15,24,25,37]$. In this method, the trial function simply converts the system of partial differential equations into a set of differential algebraic equations (DAEs). The system obtained can be solved by using any computer subroutine.

After applying the mixed orthogonal collocation on (7), following set of DAEs is obtained:

$$
\begin{gathered}
\frac{1}{\mathrm{Pe}} \sum_{i=0}^{N} B_{j i} C_{i}-\sum_{i=0}^{N} A_{j i} C_{i}=\frac{d C_{j}}{d \tau^{*}}, \quad j=1,2, \ldots, N-1 ; \\
C_{0}-\frac{1}{\operatorname{Pe}} \sum_{i=0}^{N} A_{j i} C_{i}=0, \quad j=0 ; \\
\sum_{i=0}^{N} A_{j i} C_{i}=0, \quad j=N ; \\
C_{\mathrm{av}}=\sum_{i=0}^{N} W_{i} C_{i} .
\end{gathered}
$$

The first- and second-order derivatives $\left(A_{j i} \mathrm{~s}\right.$ and $\left.B_{j i} \mathrm{~s}\right)$ are calculated by the method given by $[24,37]$. The set of DAE's is solved using MATLAB with ode15s system solver. This program uses the backward differentiation formula to solve the stiff system of differential algebraic equations. 
3.1. Collocation Points. In the present study, zeros of shifted Chebyshev polynomials have been used as collocation points, which tend to minimize the error as proposed by Fan et al. [38] and yield satisfactory results for the unsymmetrical boundary value problems. The equidistant spacing in the collocation points is usually not preferred due to Runge divergence phenomenon (Villadsen and Stewart[15]). The $N+1$ interpolation points are chosen to be the extreme values of an Nth order shifted Chebyshev polynomial. The zeros of shifted Chebyshev polynomials are calculated using GaussLobatto quadrature formula as

$$
\cos ^{-1}\left(2 \xi_{j}^{*}-1\right)=\frac{\pi(N-j)}{N}, \quad j=0,1, \ldots, N
$$

five to nineteen zeros are used as collocation points to solve the model.

3.2. Convergence Criterion. The convergence of the method for steady state is checked by applying the following formula:

$$
L^{*}=\left(\Delta x_{\ell}\right)^{2}\left\|M^{-1}\right\| C, \quad \text { with } 0<L^{*}<1,
$$

where $M$ is the lumped coefficient matrix and $C$ is the column matrix of the collocation solutions at any time $t$. For large values of the parameter $\mathrm{Pe}$, the values of $C$ may fluctuate. This indicates that more elements should be inserted to make $0<L^{*}<1$. With the increase in number of elements, the method converges asymptotically (Arora et al. [24]).

\section{Verification of the Model}

The numerical results are verified by comparing with the analytic ones given by Brenner [1]. A good agreement is found between these two results. The comparison is presented for 17 interior collocation points in Figure 2 for Pe 0, 32, and 80. A deviation of $0 \%-5 \%$ is found between the numerical results and analytic results for these Peclet numbers. However, the magnitude of error has decreased with the increase in collocation points, which is discussed in the next section.

4.1. Error Calculation. The relative error for exit solute concentration is calculated using the formulae $\left(C_{\mathrm{ex}}-C_{\mathrm{nm}}\right) / C_{\mathrm{ex}}$, where $C_{\mathrm{ex}}$ is the analytic value reported by Brenner [1] and $C_{\mathrm{nm}}$ is the numerical value of the present case for exit solute concentration. The relative error is plotted for different degrees of freedom for $\mathrm{Pe}=40$ in Figure 3 . For small degree of freedom, that is, for three interior collocation points, the error increases very sharply, but as the number of collocation points increases up to seventeen, the error becomes negligible. After seventeenth degree of freedom, the concentration profiles overlap with each other and yield identical results. It justifies the fact that with the increase in the number of interior collocation points after 15, no significant change in the relative error is observed. Also for small or medium range of $\mathrm{Pe}$, the relative error is approaching to zero as the analytic and numerical results are matching perfectly in Figure 2.

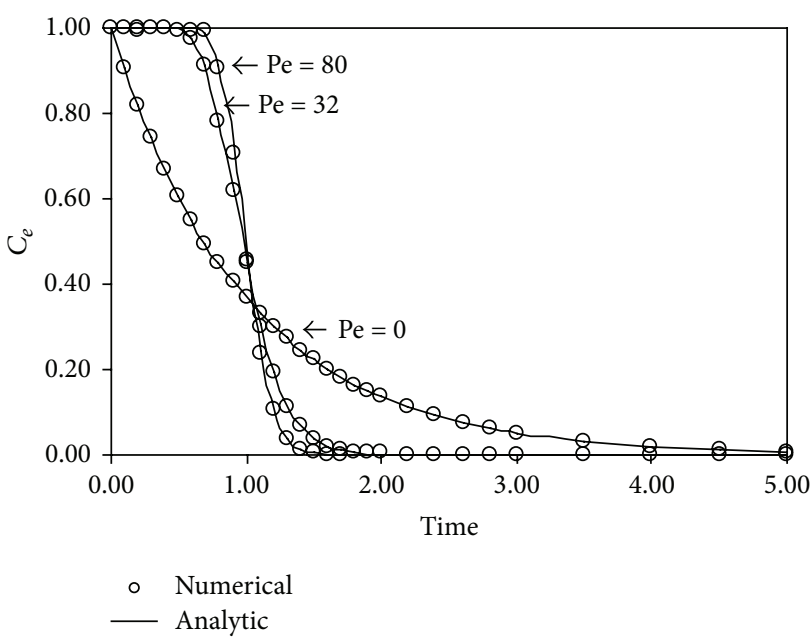

FIGURE 2: Comparison between analytic and numerical results for different Pe.

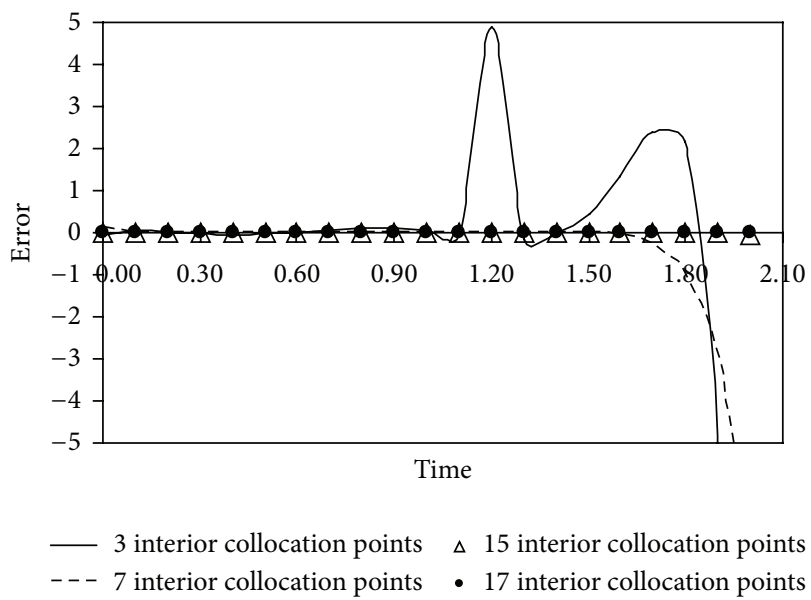

FIGURE 3: Relative error for various collocation points.

\section{Results and Discussion}

The analytic solutions of similar type of axial dispersion models using different boundary conditions are available in the literature $[1,3]$. These analytic solutions have constituted a basis for the comparison with the numerical solutions obtained from the present study. The results are presented in the form of breakthrough curves for various degrees of freedom for a wide range of Peclet numbers varying from very small to very large.

5.1. Effect of Peclet Number on $C_{a v}$. Figure 4 shows the effect of different Pe varying from 0 to 200 on average solute concentration. For $\mathrm{Pe}=200$, the concentration profile is very peaked and the convergence rate is very fast. However, for small Pe, the concentration profile is much curved and the convergence rate is very slow. It is also very clear from Figure 4 that for large $\mathrm{Pe}$, the behavior of concentration profile is more or less the same, which ultimately shows that 


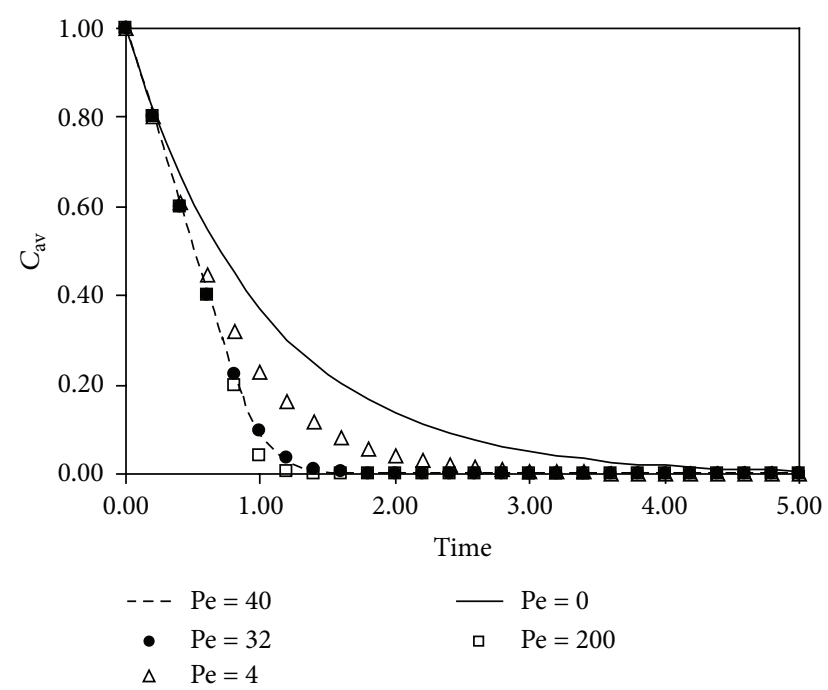

Figure 4: Effect of different Peclet numbers on $C_{\mathrm{av}}$.

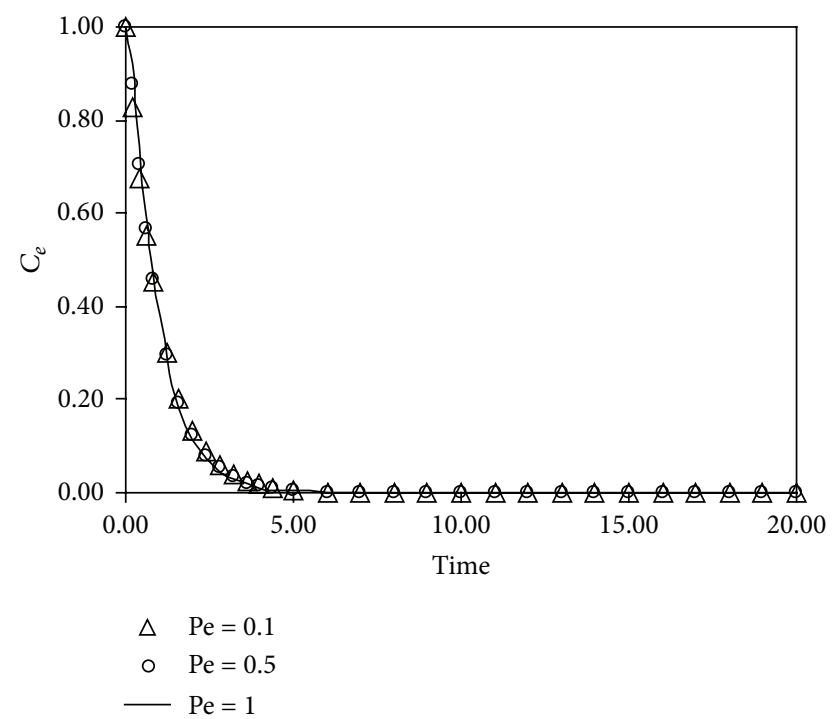

Figure 5: Effect of very small Peclet numbers on $C_{e}$.

for higher Pe the effect of axial dispersion coefficient is the least.

5.2. Effect of Peclet Number on $C_{e}$. Figure 5 shows that for very small $\mathrm{Pe}$, the exit solute concentration profile is very steep, indicating that large time period is required for the solute to diffuse out from the particle pores. As the $\mathrm{Pe}$ increases the curve follows a Gaussian shape (Figure 6). For medium range of $\mathrm{Pe}$, however, the time for washing is large resulting in slow convergence of concentration profiles, but it is still in the range of acceptability.

Figure 7 shows the effect of large Pe on exit solute concentration. It is observed that as Pe increases, the time for washing decreases sharply. It indicates the fact that for large $\mathrm{Pe}$, axial dispersion coefficient becomes smaller, as a result, more solute will diffuse out from the particle pores.

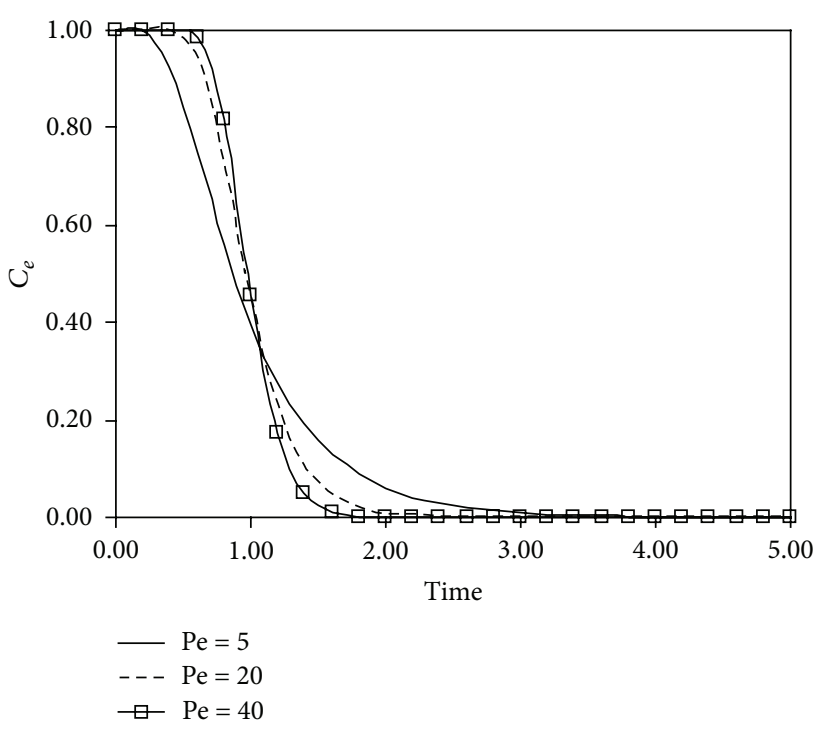

Figure 6: Gaussian behavior of $C_{e}$ curves for various Pe.

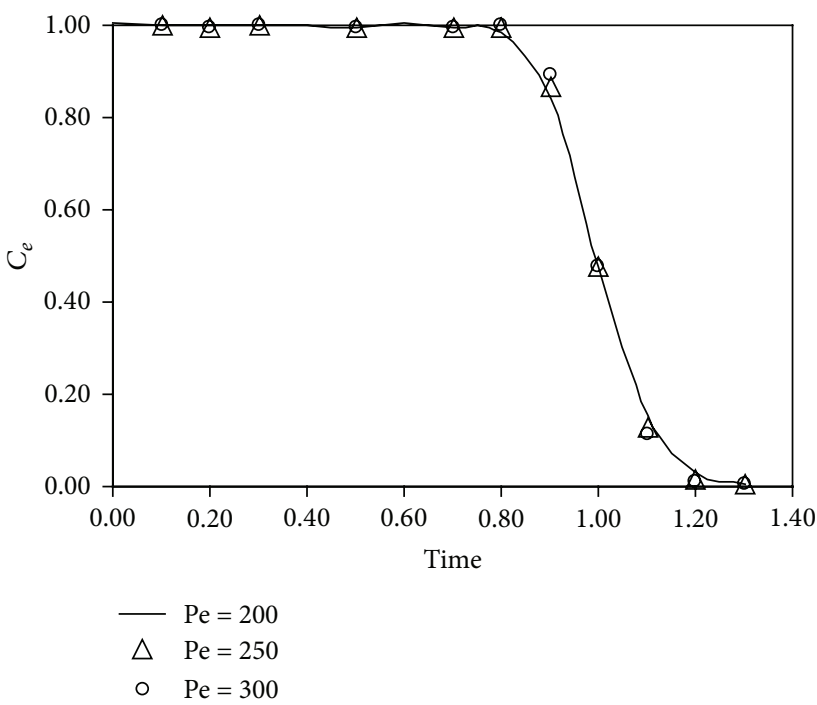

Figure 7: Effect of large Peclet numbers on $C_{e}$.

5.3. Limiting Cases. Perfect mixing, that is, when $\mathrm{Pe} \rightarrow 0$. In such situation each differential element of the solvent introduced into the bed instantaneously mixes with the contents of the bed, and an equal volume of the fluid is displaced from the bed. The bed behaves like a perfect mixing chamber. For very small $\mathrm{Pe}$, diffusion plays a dominant role since interstitial velocity is very small, resulting in slow convergence of the concentration profiles as shown in Figure 8. This type of situation is not considered ideal for industrial practice.

Perfect displacement, that is, when, $\mathrm{Pe} \rightarrow \infty$. In such a situation, (3) reduces into a partial differential equation of order one. The role of interstitial velocity becomes more important than that of the diffusion. The solution profile becomes very broaden, and time for washing falls very rapidly as shown in Figure 9. In such a situation the initial contents 


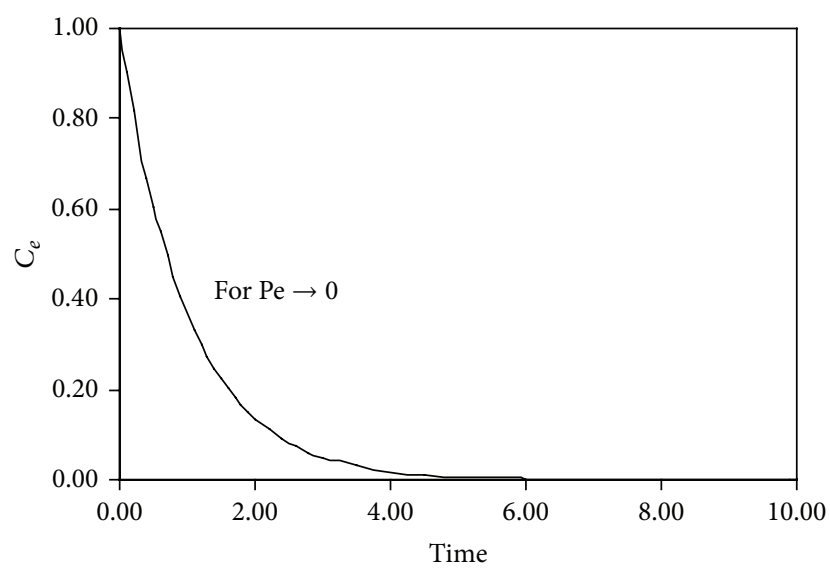

FIGURE 8: The case of perfect mixing.

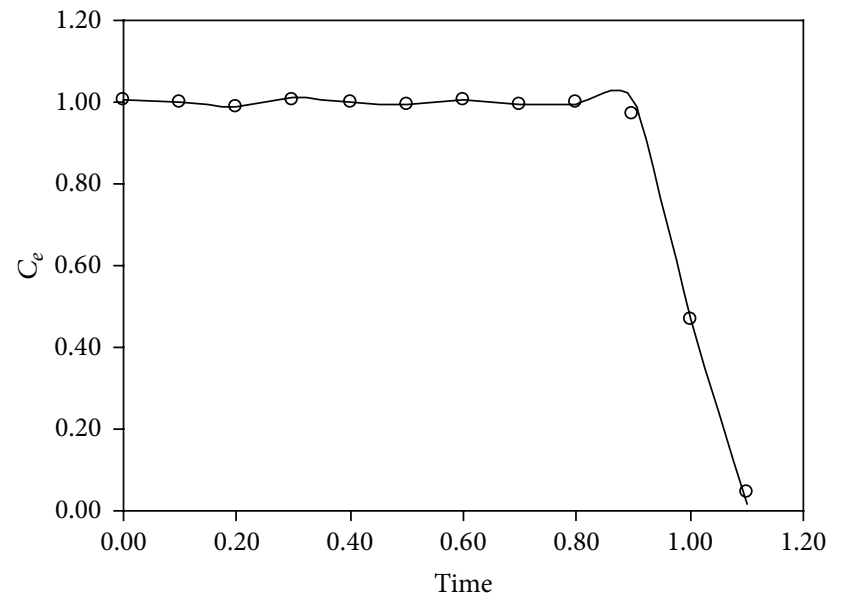

○ $\mathrm{Pe}=640$

- $\mathrm{Pe}=1000$

Figure 9: The case of perfect displacement.

of the bed are pushed out in a piston-like fashion by the displacing fluid. But this situation is not practical as the diffusion can never be zero or the interstitial velocity can never be infinite.

Ideally, it is not possible to remove all the soluble impurities from the packed bed. Therefore an optimum range of Pe should be followed in the industry to keep a balance between the removal of impurities and time of washing. AlJabari et al. [20] have also shown that flow characterization of homogeneous packed beds for bed void fraction of about 0.9 can best be represented by $\mathrm{Pe}=40$. This flow is intermediate between the cases of perfect displacement $(\mathrm{Pe}=\infty)$ and perfect mixing $(\mathrm{Pe}=0)$.

5.4. Effect of Peclet Number on Packed Bed. Effect of Pe on exit solute concentration at different locations in the mass transfer zone (MTZ) for constant $\tau^{*}$ is presented in Figure 10. For small Pe, more back mixing effect is obtained due to increasing effect of axial dispersion coefficient keeping

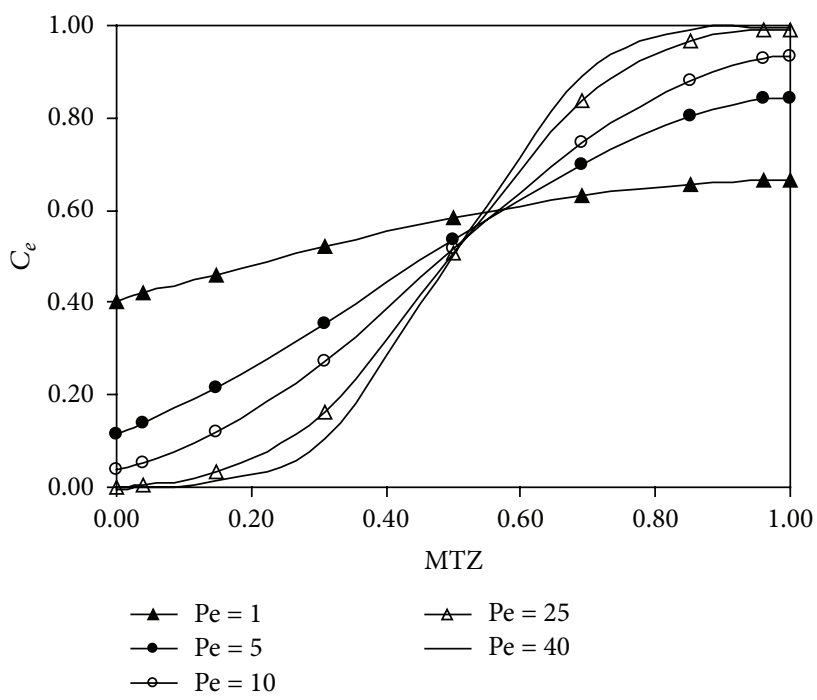

Figure 10: Effect of different Pe on mass transfer zone for fixed value of $\tau^{*}$.

the interstitial velocity and cake thickness constant. For higher Pe, steeper mass transfer zone is observed. This figure indicates that for small Pe, large time is required for the solute to diffuse out of the particle pores.

\section{Conclusions}

Numerical solution of a packed bed comprising of porous semisolid cylindrical particles is presented. A robust and convenient technique of mixed collocation method shows good agreement with the analytic results. The method is computationally efficient as it consumes less computation time. It is also observed that the efficiency of the results increases with the increase in collocation points. For efficient washing operations, the Pe should lie within the range of 20 to 40. A similar type of nonlinear problem can also be solved in exactly the same manner, as the condition of linearity is of no special use.

\section{Nomenclature}

c: Concentration of solute in the liquor, $\mathrm{kg} / \mathrm{m}^{3}$

$C:$ Dimensionless concentration, $\left(c-C_{S}\right) /\left(C_{i}-C_{S}\right)$

$C_{i}$ : Inlet solute concentration, $\mathrm{kg} / \mathrm{m}^{3}$

$C_{S}$ : Solute concentration in the wash liquor, $\mathrm{kg} / \mathrm{m}^{3}$

$D_{L}$ : Longitudinal dispersion coefficient, $\mathrm{m}^{2} / \mathrm{s}$

$k$ : Mass transfer coefficient, $\mathrm{m} / \mathrm{s}$

L: Thickness of the cake, $m$

Pe: Peclet number, dimensionless $\mathrm{Pe}=u L / D_{L}$

$q$ : Concentration of solute on the particles, $\mathrm{kg} / \mathrm{m}^{3}$

Q: Dimensionless concentration, $\left(q-C_{S}\right) /\left(C_{i}-C_{S}\right)$

$u$ : Interstitial velocity through bed, $\mathrm{m} / \mathrm{s}$

$\varepsilon$ : Porosity of cake, dimensionless

$\xi$ : Distance from point of introduction of solvent, $\mathrm{m}$ 
$\xi^{*}:$ Dimensionless distance, $\xi / L$

$\tau$ : Time, $s$

$\tau^{*}$ : Dimensionless time, $\tau^{*}=u \tau \varepsilon / L(1-\varepsilon) k$.

\section{Acknowledgments}

Ishfaq Ahmad Ganaie is thankful to NBHM, Mumbai, for JRF under the Research Project (no. 2/48(14)/2009/R\&DII/2806). Shelly Arora is thankful to UGC for providing financial assistance in the form of Research Project no. 41786/2012 (SR).

\section{References}

[1] H. Brenner, "The diffusion model of longitudinal mixing in beds of finite length. Numerical values," Chemical Engineering Science, vol. 17, no. 4, pp. 229-243, 1962.

[2] G. Eriksson, Displacement washing in the sulfate pulp mill [Ph.D. thesis], Chalmers University of Technology, Göteborg, Sweden, 1996.

[3] H. T. Liao and C. Y. Shiau, "Analytical solution to an axial dispersion model for the fixed-bed adsorber," Journal of American Institute of Chemical Engineers, vol. 46, no. 6, pp. 1168-1176, 2000.

[4] M. K. Szukiewicz, "New approximate model for diffusion and reaction in a porous catalyst," AIChE Journal, vol. 46, no. 3, pp. 661-665, 2000.

[5] F. Potůĉek, "Displacement washing of pulp II: analysis of laboratory data," Papir a Celulóza, vol. 56, no. 2, pp. 49-53, 2001.

[6] C. R. F. Pacheco, J. L. De Paiva, and A. S. Reynol Jr., "Operational evaluation of rotary drum vacuum filters for brownstock washing using basic filtration parameters," Tappi Journal, vol. 5, no. 3, pp. 15-20, 2006.

[7] V. K. Kukreja and A. K. Ray, "Mathematical modeling of a rotary vacuum washer used for pulp washing: a case study of a lab scale washer," Cellulose Chemistry and Technology, vol. 43, no. 1-3, pp. 25-36, 2009.

[8] A. La Rocca, A. H. Rosales, and H. Power, "Radial basis function Hermite collocation approach for the solution of time dependent convection-diffusion problems," Engineering Analysis with Boundary Elements, vol. 29, no. 4, pp. 359-370, 2005.

[9] C.-S. Liu, "A two-stage Lie-group shooting method (TSLGSM) to identify time-dependent thermal diffusivity," International Journal of Heat and Mass Transfer, vol. 53, no. 21-22, pp. 48764884, 2010.

[10] G. Juncu, S. Bildea, and O. Floarea, "Steady-state multiplicity analysis of the heterogeneous axial dispersion fixed-bed reactor," Chemical Engineering Science, vol. 49, no. 1, pp. 123-130, 1994.

[11] L. M. Sun and F. Meunier, "An improved finite difference method for fixed bed multicomponent sorption," Journal of American Institute of Chemical Engineers, vol. 37, no. 2, pp. 244254, 1991.

[12] G. Gürarslan and M. Sari, "Numerical solutions of linear and nonlinear diffusion equations by a differential quadrature method (DQM)," International Journal for Numerical Methods in Biomedical Engineering, vol. 27, no. 1, pp. 69-77, 2011.
[13] A. H. Khater, R. S. Temsah, and M. M. Hassan, "A Chebyshev spectral collocation method for solving Burgers'-type equations," Journal of Computational and Applied Mathematics, vol. 222, no. 2, pp. 333-350, 2008.

[14] A. Korkmaz, "Shock wave simulations using sinc differential quadrature method," International Journal of Computer Aided Engineering and Technology, vol. 28, no. 6, pp. 654-674, 2011.

[15] J. V. Villadsen and W. E. Stewart, "Solution of boundary-value problems by orthogonal collocation," Chemical Engineering Science, vol. 22, no. 11, pp. 1483-1501, 1967.

[16] L. E. Grahs, "Washing of cellulose fibre beds," Svensk Papperstidning, vol. 76, pp. 597-601, 1973.

[17] F. Shirashi, "Highly accurate solution of the axial dispersion model expressed in S-system canonical form by Taylor series method," Chemical Engineering Journal, vol. 83, no. 3, pp. 175$183,2001$.

[18] F. Liu and S. K. Bhatia, "Computationally efficient solution techniques for adsorption problems involving steep gradients in bidisperse particles," Computers and Chemical Engineering, vol. 23, no. 7, pp. 933-943, 1999.

[19] M. Xu, R.-H. Wang, J.-H. Zhang, and Q. Fang, "A novel numerical scheme for solving Burgers' equation," Applied Mathematics and Computation, vol. 217, no. 9, pp. 4473-4482, 2011.

[20] M. Al-Jabari, A. R. P. van Heiningen, and T. G. M. van de Ven, "Modeling the flow and the deposition of fillers in packed beds of pulp fibres," Journal of Pulp and Paper Science, vol. 20, no. 9, pp. J249-J253, 1994.

[21] F. Liu and S. K. Bhatia, "Application fo Petrov-Galerkin methods to transient boundary value problems in chemical engineering: adsorption with steep gradients in bidisperse solids," Chemical Engineering Science, vol. 56, no. 12, pp. 3727-3735, 2001.

[22] S. E. Onah, "Asymptotic behaviour of the Galerkin and the finite element collocation methods for a parabolic equation," Applied Mathematics and Computation, vol. 127, no. 2-3, pp. 207-213, 2002.

[23] Y. Liu and E. W. Jacobsen, "On the use of reduced order models in bifurcation analysis of distributed parameter systems," Computers and Chemical Engineering, vol. 28, no. 1-2, pp. 161-169, 2004.

[24] S. Arora, S. S. Dhaliwal, and V. K. Kukreja, "Solution of two point boundary value problems using orthogonal collocation on finite elements," Applied Mathematics and Computation, vol. 171, no. 1, pp. 358-370, 2005.

[25] S. Arora, S. S. Dhaliwal, and V. K. Kukreja, "Simulation of washing of packed bed of porous particles by orthogonal collocation on finite elements," Computers and Chemical Engineering, vol. 30, no. 6-7, pp. 1054-1060, 2006.

[26] K. Altıparmak and T. Öziş, "Numerical solution of Burgers' equation with factorized diagonal Padé approximation," International Journal of Numerical Methods for Heat \& Fluid Flow, vol. 21, no. 3-4, pp. 310-319, 2011.

[27] B. Bialecki and G. Fairweather, "Orthogonal spline collocation methods for partial differential equations," Journal of Computational and Applied Mathematics, vol. 128, no. 1-2, pp. 55-82, 2001.

[28] B. Gupta and V. K. Kukreja, "Numerical approach for solving diffusion problems using cubic B-spline collocation method," Applied Mathematics and Computation, vol. 219, no. 4, pp. 20872099, 2012.

[29] A. Sadighi and D. D. Ganji, "Exact solutions of nonlinear diffusion equations by variational iteration method," Computers 
and Mathematics with Applications, vol. 54, no. 7-8, pp. 11121121, 2007.

[30] F. Fouladi, E. Hosseinzadeh, A. Barari, and G. Domairry, "Highly nonlinear temperature-dependent fin analysis by variational iteration method," Heat Transfer Research, vol. 41, no. 2, pp. 155-165, 2010.

[31] A. R. Ghotbi, H. Bararnia, G. Domairry, and A. Barari, "Investigation of a powerful analytical method into natural convection boundary layer flow," Communications in Nonlinear Science and Numerical Simulation, vol. 14, no. 5, pp. 2222-2228, 2009.

[32] G. Domairry, A. Mohsenzadeh, and M. Famouri, “The application of homotopy analysis method to solve nonlinear differential equation governing Jeffery-Hamel flow," Communications in Nonlinear Science and Numerical Simulation, vol. 14, no. 1, pp. 85-95, 2009.

[33] M. Esmaeilpour and D. D. Ganji, “Application of He's homotopy perturbation method to boundary layer flow and convection heat transfer over a flat plate," Physics Letters A, vol. 372, no. 1, pp. 33-38, 2007.

[34] M. Omidvar, A. Barari, M. Momeni, and D. Ganji, "New class of solutions for water infiltration problems in unsaturated soils," Geomechanics and Geoengineering, vol. 5, no. 2, pp. 127-135, 2010.

[35] Z. Z. Ganji, D. D. Ganji, and M. Esmaeilpour, "Study on nonlinear Jeffery-Hamel flow by He's semi-analytical methods and comparison with numerical results," Computers \& Mathematics with Applications, vol. 58, no. 11-12, pp. 2107-2116, 2009.

[36] M. Bayat, M. Shahidi, A. Barari, and G. Domairry, "Analytical evaluation of the nonlinear vibration of coupled oscillator systems," Zeitschrift fur Naturforschung A, vol. 66, no. 1-2, pp. 67-74, 2011.

[37] B. A. Finlayson, Nonlinear Analysis in Chemical Engineering, McGraw-Hill, New York, NY, USA, 1980.

[38] L. T. Fan, G. K. C. Chen, and L. E. Erickson, "Efficiency and utility of collocation methods in solving the performance equations of flow chemical reactors with axial dispersion," Chemical Engineering Science, vol. 26, no. 3, pp. 378-387, 1971. 


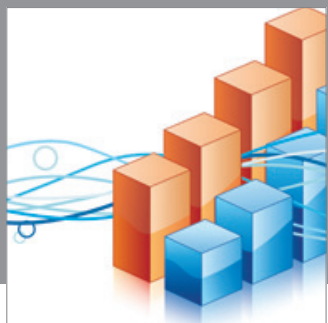

Advances in

Operations Research

mansans

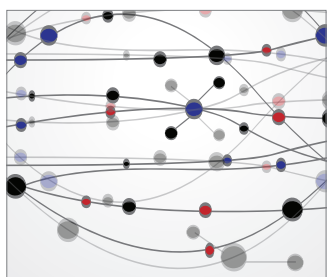

The Scientific World Journal
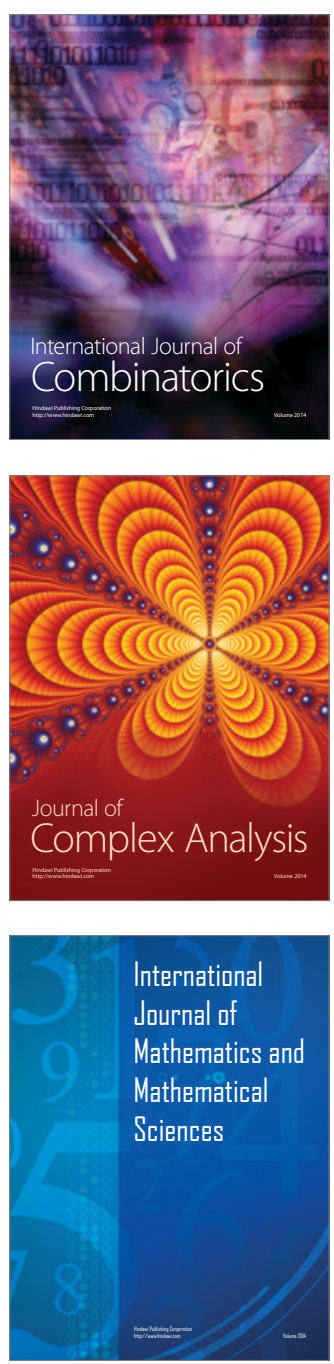
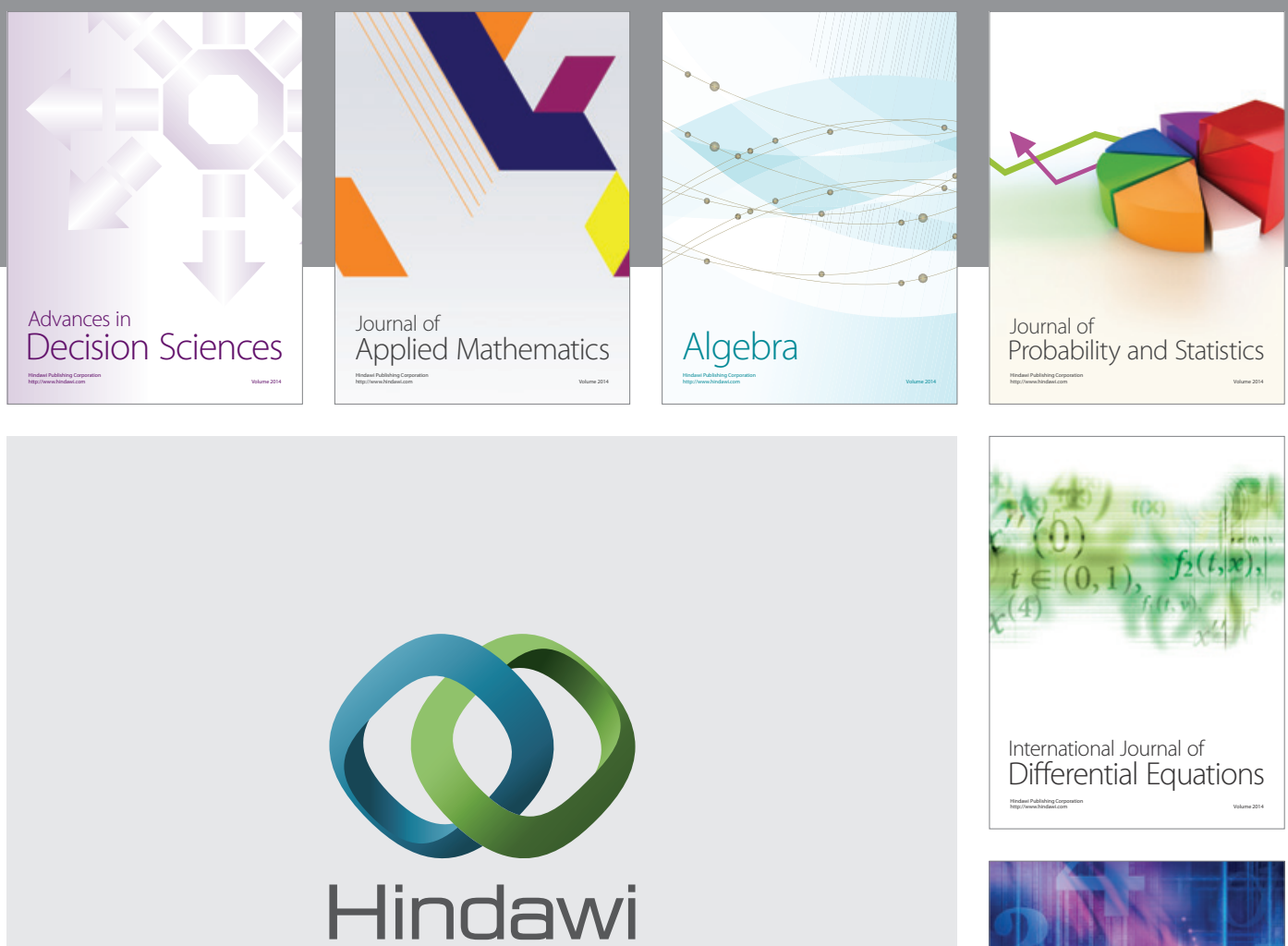

Submit your manuscripts at http://www.hindawi.com
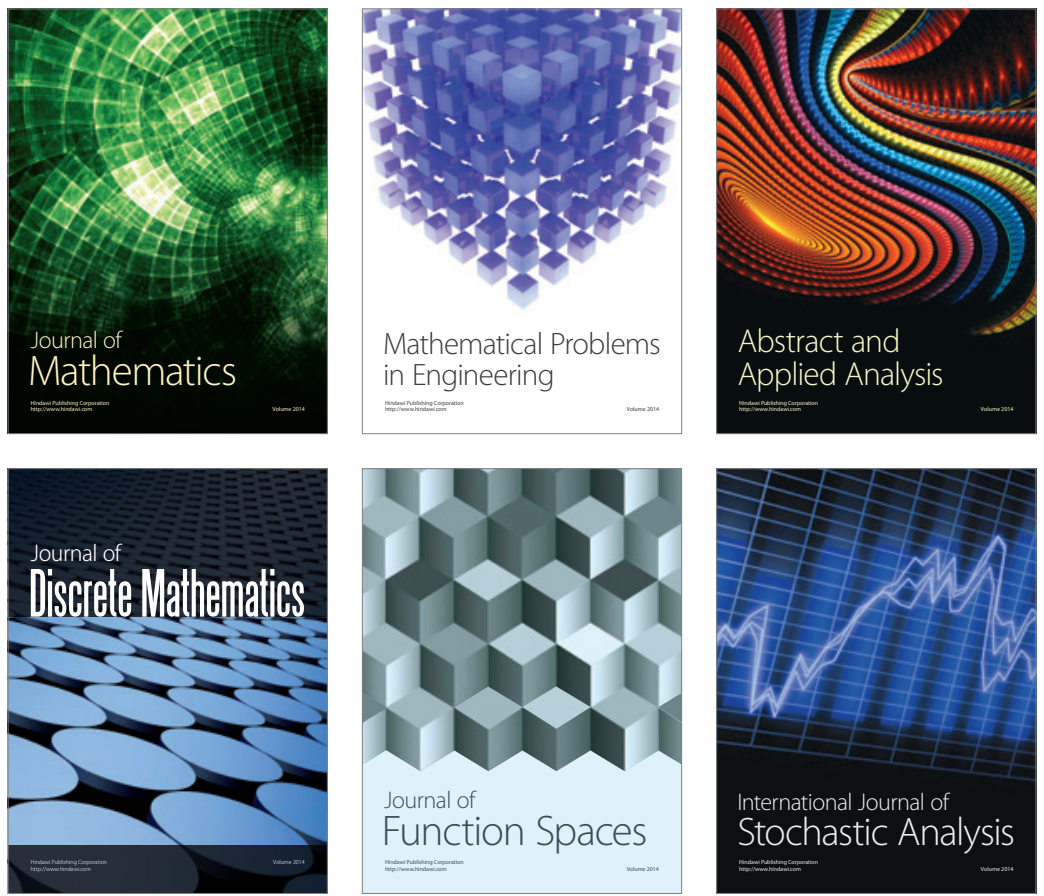

Journal of

Function Spaces

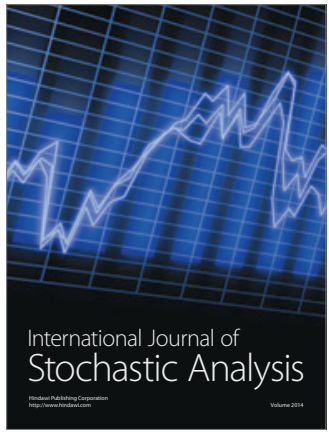

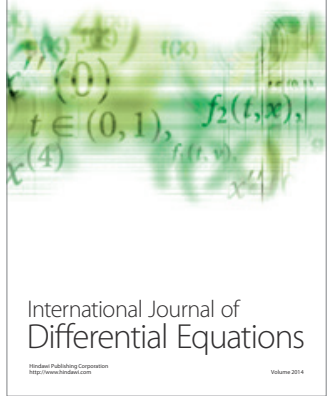
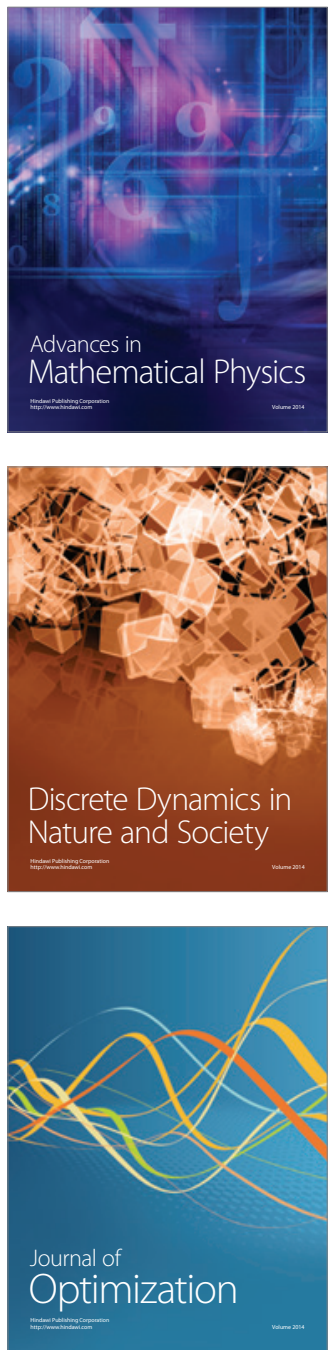